\title{
Tidal coupling of a Schwarzschild black hole and circularly orbiting moon
}

\author{
Hua Fang and Geoffrey Lovelace \\ Theoretical Astrophysics, California Institute of Technology, Pasadena, California 91125, USA
}

(Received 31 May 2005; published 16 December 2005)

\begin{abstract}
We describe the possibility of using the laser interferometer space antenna (LISA) 's gravitational-wave observations to study, with high precision, the response of a massive central body (e.g. a black hole or a soliton star) to the tidal gravitational pull of an orbiting, compact, small-mass object (a white dwarf, neutron star, or small-mass black hole). Motivated by this LISA application, we use first-order perturbation theory to study tidal coupling for a special, idealized case: a Schwarzschild black hole of mass $M$, tidally perturbed by a "moon" with mass $\mu \ll M$ in a circular orbit at a radius $b \gg M$ with orbital angular velocity $\Omega$. We investigate the details of how the tidal deformation of the hole gives rise to an induced quadrupole moment $I_{i j}$ in the hole's external gravitational field at large radii, including the vicinity of the moon. In the limit that the moon is static, we find, in Schwarzschild coordinates and ReggeWheeler gauge, the surprising result that there is no induced quadrupole moment. We show that this conclusion is gauge dependent and that the static, induced quadrupole moment for a black hole is inherently ambiguous, and we contrast this with an earlier result of Suen, which gave, in a very different gauge, a nonzero static induced quadrupole moment with a sign opposite to what one would get for a fluid central body. For the orbiting moon and the central Schwarzschild hole, we find (in agreement with a recent result of Poisson) a time-varying induced quadrupole moment that is proportional to the time derivative of the moon's tidal field, $I_{i j}=(32 / 45) M^{6} \dot{\mathcal{E}}_{i j}$ and that therefore is out of phase with the tidal field by a spatial angle $\pi / 4$ and by a temporal phase shift $\pi / 2$. This induced quadrupole moment produces a gravitational force on the moon that reduces its orbital energy and angular momentum at the same rate as the moon's tidal field sends energy and angular momentum into the hole's horizon. As a partial analog of a result derived long ago by Hartle for a spinning hole and a static distant companion, we show that the orbiting moon's tidal field induces a tidal bulge on the hole's horizon, and that the rate of change of the horizon shape (i.e. the horizon shear) leads the perturbing tidal field at the horizon by an angle $4 M \Omega$. We prefer to avoid introducing an ingoing null geodesic, as Hartle did in his definition of the phase shift, because the moon is in the central body's near zone $(b \ll 1 / \Omega)$ and thus should interact with the horizon instantaneously, not causally. We discuss the implications of these results for LISA's future observations of tidal coupling, including the inappropriateness of using the concepts of tidal polarizability and tidal lag or lead angle, for the massive central body, when discussing LISA's observations.
\end{abstract}

DOI: 10.1103/PhysRevD.72.124016

PACS numbers: 04.70.-s, 04.30.-w, 97.60.Lf

\section{INTRODUCTION AND SUMMARY}

\section{A. Motivations}

One of the primary scientific requirements for the laser interferometer space antenna (LISA) is to map, in exquisite detail, the spacetime geometries of massive black holes (and, if they exist, other massive, compact bodies) by using the gravitational waves emitted by inspiraling white dwarfs, neutron stars, and small-mass black holes. This emission process has come to be called "extreme mass ratio inspiral" (EMRI, pronounced emm-ree). The possibility of making such maps from EMRI waves was discussed by Thorne in the early 1990s (e.g., in Refs. [1,2]). In 1995 Ryan [3] laid the first detailed foundation for such mapping: he showed that, when the massive, central body is general relativistic, axisymmetric, and reflectionsymmetric, and the orbiting object is in a near-equatorial, near-circular orbit in the vacuum region surrounding the body, the full details of the central body's metric are encoded in (i) the phase evolution of the waves and also in (ii) the evolution of the frequencies (or phases) of wave modulation produced by orbital precession. Phinney [4] has given the name "bothrodesy" to the mapping of a black hole's metric via EMRI waves, and bothrodesy has been identified, by the LISA International Science Team (LIST), as one of the prime goals for LISA [5]. The initial phase of scoping out LISA's data analysis challenges for EMRI waves is now underway $[6,7]$.

Ryan's proof that the EMRI waves carry a map of the central body's metric ignored completely the influence of tidal coupling between the central body and the orbiting object. Finn and Thorne [8] have shown that, for values of the body and object masses in the range relevant to LISA, the tidal coupling can have an influence as large as a few percent on the evolution of the waves' inspiral phase-a phase that should be measurable to a fraction of a cycle out of tens or hundreds of thousands of cycles. Thus, the influence of the tidal coupling may be measurable with rather high precision. Because, in Ryan's analysis, the map is encoded redundantly in the EMRI waves' inspiral phase and in their modulations, it is reasonable to hope that the tidal coupling will break that redundancy in such a manner 
as to permit extraction of both the map and details of the central body's response to the tidal gravitational pull of the orbiting object [9].

Thorne [10] has argued that if we are to keep an open mind about the physical nature of the central body from the outset [e.g., if we are to allow for the possibility that it is a boson star (e.g. [11,12]) or a soliton star (e.g. [13]) rather than a black hole], then we must describe the tidal coupling in a manner that can encompass all possible types of central bodies - a body-independent manner.

In the case of the earth and moon, the tidal coupling is normally described in terms of the rise and fall of the earth's surface or ocean's surface, and in terms of energy dissipation in the earth's oceans. Noticeably different from this, the tidal coupling in the case of a black hole has always, until now, been described in terms of the influence of the orbiting object's gravitational field on the hole's horizon - the perturbation of the horizon's 2-metric (e.g. $[14,15])$, or the conversion of the tidal field into gravitational radiation at the horizon by gravitational blue-shifting and the energy and angular momentum carried inward by those waves (e.g., [16]).

One tidal feature in common between a black hole, the earth, a boson or soliton star, and all other conceivable central bodies, is the body's tidally-induced multipole moments and multipolar gravitational fields. It is these induced fields, acting back on the orbiting object, that change the object's orbital energy and angular momentum, secularly change its orbit, and thereby alter the emitted gravitational waves. For this reason, Thorne [10] has proposed that we adopt these induced multipole fields or moments as our body-independent description of tidal coupling when analyzing LISA data.

As a first step in exploring Thorne's proposal, we compute, in this paper, the tidally-induced quadrupole moment and its back reaction on the orbiting object, in the special case where the central body is a Schwarzschild black hole, and the object is a distant, circularly orbiting moon.

\section{B. Framework and results}

Consider a moon of mass $\mu$ orbiting around a massive central body at a large distance. When the central body is a planet [17] (see Sec. III of Ref. [15] for a review), the external tidal field produced by the moon, denoted by $\mathcal{E}_{i j}^{\text {ext }}$, raises a tide on the central body and induces a quadrupole moment $I_{i j}^{\text {ind }}$ that is proportional to $\mathcal{E}_{i j}^{\text {ext }}$. The proportionality constant is the body's polarizability. Because of viscous dissipation, the induced quadrupole moment $I_{i j}^{\text {ind }}$ will be slightly out of phase with $\mathcal{E}_{i j}^{\text {ext }}$; it will have a small phase lag with respect to the applied field. This phase lag is generally referred to as the tidal lag angle, and can be defined equivalently as the ratio of the tangential and radial component of the tidally-induced force acting back on the moon. One objective of this paper is to explore whether this type of characterization via polarizability and lag angle is also reasonable when the central body is a black hole.

To explore this, we study a model problem where the moon is orbiting circularly around a massive Schwarzschild black hole of mass $M(\gg \mu)$ at large distance $b(\gg M)$. We assume the separation $b$ is large enough that there exists an intermediate region between the hole and moon where (i) gravity is weak so space is nearly flat; (ii) the moon's tidal field does not vary appreciably. This region is referred to as the black hole's local asymptotic rest frame (LARF) [18]. Because the spacetime is nearly flat, one can write down the full tidal field in the LARF (in Cartesian coordinates) to linear order in each multipole moment as [19]

$$
\begin{aligned}
\mathcal{E}_{i j} \equiv & R_{0 i 0 j} \\
= & -\sum_{\ell=0}^{\infty} \frac{(-1)^{\ell}}{\ell !} I_{A_{\ell}}\left(\frac{1}{r}\right)_{, i j A_{\ell}} \\
& -\sum_{\ell=2}^{\infty} \frac{(2 \ell-1) ! !}{(\ell-2) !} Q_{i j A_{\ell-2}} X_{A_{\ell-2}} .
\end{aligned}
$$

Here $I_{A_{\ell}}$ and $Q_{A_{\ell}}$ are the $\ell^{\prime}$ th internal and external moments; they are symmetric and trace-free (STF) in their tensor indices $A_{\ell} \equiv a_{1} \ldots a_{\ell}$ [20]. The "internal moments" $I_{A_{\ell}}$ characterize the central body, while the "external moments" $\mathcal{Q}_{A_{\ell}}$ characterize the gravitational fields of distant sources that perturb the central body. In our problem, the tidal field $\mathcal{E}_{i j}^{\text {ext }}$ is physically the same as the external quadrupole moment $\mathcal{Q}_{i j}$; they differ only by a constant scaling factor, $\mathcal{E}_{i j}^{\mathrm{ext}}=-3 \mathcal{Q}_{i j}$. The internal quadrupole moment is induced by the applied tidal field and characterizes the tidal deformation of the central body.

Equation (1) is the gravitational analogy to the multipole expansion of an electromagnetic field. It will be sufficiently accurate for our purpose, since we shall compute the nonspherical parts of the Riemann curvature tensor by solving the linearized Einstein field equations. It will be shown in Sec. II that only multipole moments with $\ell=0,2$ are relevant to our problem. Dropping all other terms in Eq. (1) and contracting with the unit spatial vector yields

$$
\mathcal{E}_{i j} n^{i} n^{j}=-\frac{2 M}{r^{3}}+\mathcal{E}_{i j}^{\mathrm{ext}} n^{i} n^{j}-\frac{18 I_{i j}^{\mathrm{ind}} n^{i} n^{j}}{r^{5}},
$$

where we have identified $I$ in Eq. (1) as the total mass of the black hole and substituted the external tidal field $\mathcal{E}_{i j}^{\text {ext }}$ for $\mathcal{Q}_{i j}$. In the last term, $I_{i j}^{\text {ind }}$ represents the quadrupole moment induced on the black hole by the external tidal field.

In Secs. III and IVA of this paper we compute the induced quadrupole moment in Regge-Wheeler gauge, obtaining

$$
I_{i j}^{\text {ind }}=\frac{32}{45} M^{6} \dot{\mathcal{E}}_{i j}^{\mathrm{ext}}
$$


The same result was recently derived by Poisson from calculating the averaged rate of change of mass and angular momentum of the perturbed black hole [21]. Note that $I_{i j}^{\text {ind }}$ is proportional to the time derivative of $\mathcal{E}_{i j}^{\text {ext }}$ (a time derivative caused by the moon's motion) and is therefore completely out of phase with the external tidal field (by 90 degrees in time and 45 degrees in space). As we will show in Sec. V, this out-of-phase induced moment is gauge invariant and is responsible for the torque that changes the orbital energy and angular momentum. Thus it is also responsible for the tidally-induced portion of the orbital evolution and the phase evolution of the gravitational waves.

The piece of the induced quadrupole moment that is proportional to and in-phase with the applied tidal field is ambiguous (in a sense that we shall discuss in Sec. IV B); in Schwarzschild coordinates and Regge-Wheeler gauge, it vanishes. If there had been an unambiguous piece of $I_{i j}^{\text {ind }}$ in phase with $\mathcal{E}_{i j}^{\text {ext }}$, then this in-phase piece would have defined a polarizability, and the ratio of out-of-phase piece to the in-phase piece would have been, in a certain welldefined sense, the small tidal lag angle. Thus, our result can be regarded as saying that both the polarizability and the lag angle of a black hole are ambiguous (in the sense discussed in Sec. IV B).

Although we find that the tidal lag angle in the LARF, in the case of a Schwarzschild black hole, is ambiguous, we can still define and calculate an angular tidal shift on the horizon (as opposed to in the LARF or out at the moon). We study this horizon phase shift in Sec. V. Hartle [15] has calculated $^{1}$ the tidal lag angle for the problem of a bulge raised on slowly rotating hole's horizon by a stationary moon, and he has shown it to be negative: the horizon's tidal bulge leads the applied tidal field due to the horizon's teleological definition (i.e., a definition in terms of the future fate of null rays). As in Hartle's case, we can compare the phase of the shape of our nonrotating horizon to our moving moon's position by mapping the moon to the horizon with an ingoing, zero-angular momentum, null geodesic. In Sec. V, we find that this prescription leads to a lead angle between the moon and the horizon

$$
\delta_{\text {null map }}=\frac{8}{3} M \Omega+\Omega b^{*}
$$

where $\Omega$ is the orbital angular frequency of the moon and $b^{*}$ is the moon's tortoise coordinate $b^{*} \equiv b+$ $2 M \log (b / 2 M-1)$.

For comparison, Hartle's result [15] for the tidal lead angle in the case of the rotating hole and distant, stationary moon in the equatorial plane, is (after correcting a sign error, as discussed in footnote ${ }^{6}$ )

\footnotetext{
${ }^{1}$ We review the principal results of Hartle's investigation in Sec. VB 1.
}

$$
\delta_{\text {null map }}^{\mathrm{H}}=\frac{2 a}{3 M}+\frac{a}{b}=\frac{8}{3} M \Omega_{H}+4 \frac{M^{2} \Omega_{H}}{b},
$$

Here $a$ is the hole's specific angular momentum, and $\Omega_{H}$ is the horizon angular velocity. The radius of the moon's position $b$ is sufficiently large that the moon is essentially stationary. Throughout this paper, we use the superscript " $\mathrm{H}$ " to indicate results corresponding to Hartle's system, i.e., to a system with a stationary moon and rotating horizon. Other results (without the subscript " $\mathrm{H}$ ") correspond to our system of a distant moon, orbiting at frequency $\Omega$, which perturbs a Schwarzschild black hole).

Our result (4) differs from Hartle's (5) — even though we initially expected that the tidal phase shift should depend only on the difference in angular velocities of the applied tidal field and the horizon generators, so the results would be the same. The terms that differ arise from the particular choice to map the moon to the horizon using an ingoing, zero-angular momentum null ray.

We prefer an alternative definition of the tidal lead angle, one that is independent of $b^{*}$; we prefer to define the tidal phase shift as the angle between the perturbing tidal field at the horizon and the shear (which is the rate of change of the shape) of the horizon [22]. This definition avoids introducing null connections between the moon (which, at radius $b \ll \Omega^{-1}$, is in the near zone) and the horizon. Using this definition, we find that the shear of the central hole leads the perturbing tidal field at the horizon by an angle

$$
\delta_{\text {Horizon }}=\delta_{\text {Horizon }}^{\mathrm{H}}=4 M \Omega .
$$

The tidal lead angle is the same whether one considers a stationary moon perturbing a rotating hole or an orbiting moon perturbing a nonrotating hole.

The rest of paper is organized as follows. In Sec. II, we decompose the applied tidal field in the LARF into a timedependent part and a static part. In Sec. III we analyze fully the time-dependent part and deduce the dynamical part of the induced quadrupole moment [Eq. (24)]. In Sec. IV, we solve for the static perturbation and discuss the ambiguity in defining the static part of the induced quadrupole moment. In Sec. V, we study the phase shift between the deformation of the horizon and the applied tidal and compare the result with the phase shift as defined by Hartle. A brief conclusion is made in Sec. VI. Throughout the paper, we use geometrized units with $G=c=1$.

\section{PROBLEM SETUP}

We study small perturbations of a nonspinning black hole caused by an orbiting moon. The unperturbed background metric is the Schwarzschild metric:

$$
\begin{aligned}
d s^{2}= & -\left(1-\frac{2 M}{r}\right) d t^{2}+\frac{d r^{2}}{1-2 M / r} \\
& +r^{2}\left(d \theta^{2}+\sin ^{2} \theta d \phi^{2}\right),
\end{aligned}
$$


where $M$ is the mass of the central hole. At large radii (i.e., in the LARF), we will study the perturbations in a notation that treats the Schwarzschild coordinates $(r, \theta, \phi)$ as though they were flat-space spherical coordinates. These coordinates are related to the Cartesian coordinates $(x, y, z)=\left(x^{1}, x^{2}, x^{3}\right)$ by

$$
\left(x^{1}, x^{2}, x^{3}\right)=r(\sin \theta \cos \phi, \sin \theta \sin \phi, \cos \theta) .
$$

We will denote the radial vector with length $r$ by $\mathbf{x}$, the unit radial vector by $\mathbf{n}$, and their components by $x^{j}$ and $n^{j}$, respectively.

Let a moon of mass $\mu$ move along a circular orbit with radius $b$ in the equatorial plane $(b \gg M \gg \mu)$. The moon's position is specified by

$$
\mathbf{x}^{\mathrm{s}}=b \mathbf{n}^{\mathrm{s}}=b(\cos \Omega t, \sin \Omega t, 0),
$$

where the superscript "s" stands for the "source" of the perturbation and $\Omega=\sqrt{M / b^{3}}$ is the moon's orbital angular frequency, satisfying $\Omega b \ll 1$. The moon's tidal field $\mathcal{E}_{i j}^{\text {ext }}$ is the double gradient of the moon's Newtonian gravitational potential. Its value in the LARF (at $r \ll b$ but $r \gg$ $M)$ is well approximated by

$$
\mathcal{E}_{i j}^{\mathrm{ext}}=-\left.\left(\frac{\mu}{\left|\mathbf{x}-\mathbf{x}^{\mathrm{s}}\right|}\right)_{, i j}\right|_{r=0} \quad=\frac{\mu}{b^{3}}\left(\delta_{i j}-3 n_{i}^{\mathrm{s}} n_{j}^{\mathrm{s}}\right) .
$$

Note that although the applied tidal field is defined in the LARF, the induced quadrupolar field $I_{i j}^{\text {ind }}$ of greatest interest is not in the LARF, but further out in the vicinity of the moon's orbit, where it interacts with the moon.

The tidal field (9) can be decomposed into spherical, harmonic modes [23]. The result of the decomposition is

$$
\begin{aligned}
\mathcal{E}_{i j}^{\mathrm{ext}} & =\frac{\mu}{b^{3}} \sqrt{\frac{6 \pi}{5}}\left(\sqrt{\frac{2}{3}} \mathcal{Y}_{i j}^{20}-\mathcal{Y}_{i j}^{22} e^{-i \omega t}-\mathcal{Y}_{i j}^{2-2} e^{i \omega t}\right) \\
& \equiv \mathcal{E}_{i j}^{\mathrm{ext}, 20}+\mathcal{E}_{i j}^{\mathrm{ext}, 22}+\mathcal{E}_{i j}^{\mathrm{ext}, 2-2}
\end{aligned}
$$

with $\omega \equiv 2 \Omega$ and $\mathcal{E}_{i j}^{\text {ext,2m }}(m=0$, \pm 2$)$ equal to the corresponding $\mathcal{Y}_{i j}^{2 m}$ term. Here the $\mathcal{Y}_{i j}^{2 m}$ are positionindependent, rank-2, symmetric trace-free (STF) tensors defined in Eqs. (A3) and (A4) and are related to the familiar $\ell=2$ spherical harmonics $Y^{2 m}(\theta, \phi)$ by Eq. (A2). (See Eqs. (2.7)-(2.14) of Ref. [23] for the general mapping between order $\ell$ spherical harmonics and rank- $\ell$ STF tensors.) The explicit values of the tidal field compo-

nents are

$$
\begin{aligned}
\mathcal{E}_{i j}^{\mathrm{ext}, 20} & =-\frac{\mu}{2 b^{3}}\left(\begin{array}{ccc}
1 & 0 & 0 \\
0 & 1 & 0 \\
0 & 0 & -2
\end{array}\right), \\
\mathcal{E}_{i j}^{\mathrm{ext}, 2 \pm 2} & =-\frac{3 \mu}{4 b^{3}}\left(\begin{array}{ccc}
1 & \pm i & 0 \\
\pm i & -1 & 0 \\
0 & 0 & 0
\end{array}\right) e^{\mp i \omega t}
\end{aligned}
$$

The tidal field $\mathcal{E}_{i j}^{\text {ext }}$ [Eq. (10)] is the source of perturbations of the central hole; it is an even-parity $\ell=2$ external tidal field. We shall therefore perform our calculation in the even-parity Regge-Wheeler gauge, mode by mode $(\ell=$ $2, m=0, \pm 2)$. The tidal field $\mathcal{E}_{i j}^{\text {ext }}$ also sets the outer boundary condition for the problem: the $O\left(r^{0}\right)$ terms in the perturbed tidal field $\mathcal{E}_{i j}$ must go to $\mathcal{E}_{i j}^{\text {ext }}$ in the LARF [Eq. (2)].

The inner boundary condition is set differently, depending on whether the perturbations are static or timedependent. For the static perturbations generated by $\mathcal{E}_{i j}^{20}$, we impose a "regularity boundary condition": the perturbations must be physically finite at $r=2 M$. For the timedependent perturbations generated by $\mathcal{E}_{i j}^{\text {ext,2 } 2 \pm 2}$, we impose the "ingoing-wave boundary condition": the perturbations have the asymptotic behavior $\sim e^{\mp i \omega r^{*}}$ when approaching the horizon. Here $r^{*}$ is the tortoise coordinate $r^{*} \equiv r+$ $2 M \log (r / 2 M-1)$.

\section{TIME-DEPENDENT PART OF THE PERTURBATION}

\section{A. The perturbed metric}

We will specialize to $(\ell, m)=(2,2)$ in solving for the time-dependent part of the metric perturbation. The $(\ell, m)=(2,-2)$ results can be obtained by complex conjugating the $(2,2)$ results. For briefness, a superscript " 22 " will not be added to quantities calculated in this harmonic mode in this section, unless a distinction is needed. Throughout this section, we refer to Appendix B for details of the perturbation calculation.

In the standard Regge-Wheeler gauge, the $(\ell, m)=$ $(2,2)$ time-dependent perturbations take the form [24]

$$
h_{a b}^{(22)}=Y^{22}(\theta, \phi) e^{-i \omega t}\left|\begin{array}{cccc}
H\left(1-\frac{2 M}{r}\right) & H_{1} & 0 & 0 \\
H_{1} & H\left(1-\frac{2 M}{r}\right)^{-1} & 0 & 0 \\
0 & 0 & r^{2} K & 0 \\
0 & 0 & 0 & r^{2} K \sin ^{2} \theta
\end{array}\right| \text {. }
$$

Here $H, H_{1}$, and $K$ are functions of $r$ alone. These radial functions are solutions of the perturbed Einstein equations; they can be constructed from the Zerilli function $\mathbb{Z}(r)$ [25], which satisfies a second-order ordinary differential equation 
[Eq. (B4)]. Specifically, $H_{1}, K$ and $H$ are given in terms of $\mathbb{Z}(r)$ by Eqs. (B1)-(B3). Instead of solving for $\mathbb{Z}(r)$ directly, one may obtain the Zerilli function from its oddparity correspondent, the Regge-Wheeler function $\mathbb{X}(r)$, which obeys a simpler differential equation $[24,26]$ that is easier to solve [Eq. (B5)]:

$$
\left[\frac{d^{2}}{d r^{* 2}}+\omega^{2}-\left(1-\frac{2 M}{r}\right)\left(\frac{\ell(\ell+1)}{r^{2}}-\frac{6 M}{r^{3}}\right)\right] \mathbb{X}(r)=0,
$$

where $d / d r^{*}=(1-2 M / r) d / d r$. The Zerilli function $\mathbb{Z}(r)$ is expressed in terms of $\mathbb{X}(r)$ by Eq. (B6). Thus, the metric perturbation is determined by the single radial function $\mathbb{X}(r)$, by way of Eq. (B6) to get $\mathbb{Z}(r)$ and then Eqs. (B1)-(B3) to get $H_{1}, K$, and $H$.

The analytic solution for $\mathbb{X}(r)$ with the ingoing-wave boundary condition at horizon was derived by Poisson and Sasaki [27]. Their solution, $X^{H}$ in their notation and for the limiting case $\omega r \ll 1$, is what we have used in our analysis. With our slow motion assumption $\Omega b \ll 1, X^{H}(\omega r \ll$ 1) will be sufficient to cover the region inside the moon's orbit - including the LARF, where we read out the induced quadrupole moment. Following Poisson and Sasaki's notation, we define the dimensionless quantity

$$
\varepsilon \equiv 2 M \omega
$$

We then combine Eqs. (3.4), (3.11), and (3.12) of Ref. [27] to obtain

$$
\begin{aligned}
X^{H}(\omega r \ll 1)= & \mathcal{A}\left(\frac{r}{2 M}\right)^{3} e^{i \omega(r-2 M)} \\
& \times F\left(c_{1}, c_{2} ; c_{3} ; 1-\frac{r}{2 M}\right) e^{-i \omega r^{*}},
\end{aligned}
$$

where $\mathcal{A}$ is an overall scaling factor that did not appear in Ref. [27] but will be determined by the outer boundary condition in our problem; $F$ is the hypergeometric function with parameters [Eq. (3.11) of Ref. [27] with $\ell=2$ ]

$$
\begin{gathered}
c_{1}=-i \varepsilon+O\left(\varepsilon^{2}\right), \quad c_{2}=5-i \varepsilon+O\left(\varepsilon^{2}\right), \\
c_{3}=1-2 i \varepsilon .
\end{gathered}
$$

Note that expression (14) for $X^{H}$ is only accurate to first order in $\varepsilon$. We then expand Eq. (14) in large $r$ and keep terms to first order in $\varepsilon$

$$
X^{H}=\mathcal{A}\left[\left(1+\frac{13}{12} i \varepsilon\right) \tilde{r}^{3}+\sum_{n=5}^{\infty} \frac{i \varepsilon}{n \tilde{r}^{n-3}}+O\left(\varepsilon^{2}\right)\right],
$$

where $\tilde{r} \equiv r / 2 M$ is the dimensionless radius. Next, we use Eq. (B6) to get $\mathbb{Z}(r)$. Then the perturbed metric components can be constructed using Eqs. (B1)-(B3). In the following all quantities will be calculated up to first order in $\varepsilon$ and we will suppress " $O\left(\varepsilon^{2}\right)$ " in our expressions.

\section{B. Induced quadrupole moment in the LARF}

Once the perturbed metric is known, it is straightforward to calculate the full Riemann tensor and extract from it the first-order tidal field in the LARF:

$$
\mathcal{E}_{i j}^{(1)} \equiv R_{0 i 0 j}^{(1)}=R_{0 i 0 j}-R_{0 i 0 j}^{(0)},
$$

where a superscript of "(0)" or "(1)" indicates the quantity is of zeroth or first order in the perturbation. In our calculation, we found it convenient to look at the $0 \mathrm{r} 0 \mathrm{r}$ component of the first-order Riemann tensor in the LARF, since

$$
R_{0 r 0 r}^{(1)}=R_{0 i 0 j}^{(1)} n_{i} n_{j}=\mathcal{E}_{i j}^{(1)} n_{i} n_{j} .
$$

From this equation we can read off $\mathcal{E}_{i j}^{(1)}=R_{0 i 0 j}^{(1)}$, the firstorder tidal field in Cartesian coordinates in the LARF, from the Riemann tensor in Schwarzschild coordinates. By the procedure outlined in this paragraph we have deduced the following $(\ell, m)=(2,2)$ part of $\mathcal{E}_{i j}^{(1)}$ in the LARF:

$$
\mathcal{E}_{i j}^{(1), 22}=-\frac{3 \mathcal{A}}{4 M^{3}}\left[1+\frac{4}{3} i \varepsilon+\sum_{n=5}^{\infty} \frac{i \varepsilon}{n \tilde{r}^{n}}\right] \mathcal{Y}_{i j}^{22} e^{-i \omega t} .
$$

The outer boundary condition states that the $O\left(r^{0}\right)$ [i.e. $O\left(\tilde{r}^{0}\right)$ ] term of $\mathcal{E}_{i j}^{(1), 22}$ must equal $\mathcal{E}_{i j}^{\text {ext,22 }}$ [Eq. (11b)]. This determines the scaling factor to be

$$
\mathcal{A}=\frac{4 \mu M^{3}}{b^{3}} \sqrt{\frac{2 \pi}{15}}\left(1-\frac{4}{3} i \varepsilon\right) .
$$

Inserting Eq. (20) into Eq. (19), we can write $\mathcal{E}_{i j}^{(1), 22}$ as

$$
\mathcal{E}_{i j}^{(1), 22}=\mathcal{E}_{i j}^{\mathrm{ext}, 22}-\frac{\mu}{b^{3}} \sqrt{\frac{6 \pi}{5}} \sum_{n=5}^{\infty} \frac{i \varepsilon}{n \tilde{r}^{n}} \mathcal{Y}_{i j}^{22} e^{-i \omega t}
$$

Here the $O\left(1 / \tilde{r}^{5}\right)$ term, by Eq. (2), contains the induced quadrupole moment. The $O\left(1 / \tilde{r}^{6}\right)$ and higher terms are proportional to the $O\left(1 / \tilde{r}^{5}\right)$ term and contain no new information; they represent the nonlinear coupling between the induced quadrupole and the black hole's monopole moment.

Comparing the $O\left(1 / \tilde{r}^{5}\right)$ term in Eq. (2) and the $O\left(1 / r^{5}\right)$ term in Eq. (21), we find that

$$
I_{i j}^{22}=\frac{32}{45} M^{6} \dot{\mathcal{E}}_{i j}^{\mathrm{ext}, 22} .
$$

Complex conjugating this equation yields the $(\ell, m)=$ $(2,-2)$ part of the induced quadrupole moment:

$$
I_{i j}^{2-2}=\frac{32}{45} M^{6} \dot{\mathcal{E}}_{i j}^{\mathrm{ext}, 2-2} .
$$

Thus, the time-dependent part, i.e. the dynamical part (DP), of the induced quadrupole moment is given by

$$
I_{i j}^{\text {ind,DP }}=\frac{32}{45} M^{6}\left(\dot{\mathcal{E}}_{i j}^{\text {ext,22 }}+\dot{\mathcal{E}}_{i j}^{\mathrm{ext}, 2-2}\right)=\frac{32}{45} M^{6} \dot{\mathcal{E}}_{i j}^{\mathrm{ext}} .
$$


This agrees with the result recently obtained by Poisson [21] by a very different method. Note that the induced quadrupole moment is proportional to the time derivative of the applied tidal field. Hence the induced quadrupole moment and the applied tidal field are completely out of phase with each other $(\pi / 4$ phase shift in space, $\pi / 2$ in time). This leads to a dissipative force acting back on the moon.

From the induced quadrupole moment (24), we define a corresponding Newtonian potential in the LARF and out to the moon's orbit:

$$
\Phi=-\frac{3}{2} I_{i j}^{\mathrm{ind}, \mathrm{DP}} \frac{n^{i} n^{j}}{r^{3}} .
$$

Then the force acting back on the moon can be found by evaluating the gradient of $\Phi$ at the moon's position:

$$
\mathbf{F}=-\left.\nabla \Phi\right|_{\mathbf{x}^{s}}=-\frac{32}{5}\left(\frac{\mu}{b}\right)^{2}\left(\frac{M}{b}\right)^{13 / 2} \mathbf{e}_{\phi}
$$

Equation (26) shows that the force is tangential and opposite to the moon's motion. The energy loss from the moon's orbital motion is then

$$
\dot{E}=-\mathbf{F} \cdot \mathbf{v}=\frac{32}{5} M^{4} \mu^{2} \Omega^{6},
$$

where $\mathbf{v}=\Omega b \mathbf{e}_{\phi}$. It is straightforward to show that there is also an angular momentum loss of magnitude $\dot{E} / \Omega$. Equation (27) agrees with Poisson and Sasaki's calculation of the rate at which the perturbation carries energy into the black hole's horizon at the leading post-Newtonian order [27].

\section{THE STATIC, AXISYMMETRIC PART OF THE PERTURBATION}

\section{A. Static induced quadrupole moment}

We now specialize to the even-parity, static part of the moon's perturbation: $(\ell, m)=(2,0)$. The Regge-Wheeler metric for this type of perturbation has the form [24]

$$
\begin{aligned}
h_{a b}^{(20)}= & \operatorname{Diag}\left[\left(1-\frac{2 M}{r}\right) \hat{H}_{2}, \frac{\hat{H}_{2}}{1-2 M / r}, r^{2} \hat{K}_{2}, r^{2} \hat{K}_{2} \sin ^{2} \theta\right] \\
& \times Y^{20}(\theta, \phi),
\end{aligned}
$$

where "Diag" is short for diagonal matrix and $\hat{H}_{2}$ and $\hat{K}_{2}$ are functions of $r$ only. The general solution to the field equation governing $\hat{H}_{2}$ can be expressed in terms of the associated Legendre functions [14]:

$$
\hat{H}_{2}(r)=\alpha_{2} P_{2}^{2}\left(\frac{r}{M}-1\right)+\beta_{2} Q_{2}^{2}\left(\frac{r}{M}-1\right),
$$

where $\alpha_{2}$ and $\beta_{2}$ are constants to be determined. The solution to $\hat{K}_{2}$ can then be obtained from that of $\hat{H}_{2}$ (Appendix C). As $r$ approaches 2M, we have [28]

$$
Q_{\ell}^{2}(r / M-1) \sim(r / M)^{-1 / 2},
$$

so the $Q_{2}^{2}$ term in Eq. (29) becomes divergent at $r=2 M$ and we must set the coefficient $\beta_{2}$ to be zero in order for the perturbation to be finite there. As $r$ goes to infinity ${ }^{2}$,

$$
P_{\ell}^{2}(r / M-1) \sim(r / M)^{\ell} .
$$

Therefore the remaining $P_{2}^{2}$ term in Eq. (29) keeps growing quadratically as $r$ becomes large, corresponding to the nonasymptotic-flatness due to the presence of the moon.

With the metric perturbation $h_{a b}^{(20)}$, we compute the Riemann tensor from the full metric and series expand the result up to linear order in $\alpha_{2}$ (i.e., first order in the perturbation). The $0 r 0 r$ component of the resulting firstorder Riemann tensor is found to be

$$
R_{0 r 0 r}^{(1)}=\frac{3 \alpha_{2}}{M^{2}} Y^{20}(\theta, \phi) .
$$

From this and from Eq. (10), we obtain the first-order tidal field in the Cartesian basis

$$
\mathcal{E}_{i j}^{(1)}=\frac{3 \alpha_{2}}{M^{2}} \mathcal{Y}_{i j}^{20}
$$

The static, first-order tidal field thus contains only an $O\left(r^{0}\right)$ term, which should be identified as the static part of the applied external field $\mathcal{E}_{i j}^{\text {ext,20 }}$ [Eq. (11a)]. The coefficient $\alpha_{2}$ is determined from this identification to be $\alpha_{2}=$ $\sqrt{4 \pi / 45} \mu M^{2} / b^{3}$. Since there is no $O\left(1 / r^{5}\right)$ term present in Eq. (31), we infer that there is no static induced quadrupole moment:

$$
I_{i j}^{20}=0 .
$$

This is quite a counterintuitive result. It is worth pointing out, however, that the absence of negative powers of $r$ in Eq. (31) follows directly from the regularity condition we imposed at $r=2 M$. If the radius $r=2 M$ were well inside the central body itself, which naturally is the case for any nonrelativistic body with weak self-gravity, then the $Q_{2}^{2}$ term in Eq. (29) would survive and give rise to an induced quadrupole moment. Equation (32) may also be the consequence of the gauge (Regge-Wheeler) we choose to work in. Is it possible to give a gauge-invariant definition of static, induced multipole moment in a non-asymptotically-flat spacetime? This is the question we shall investigate in the next subsection.

We now summarize and conclude that the total induced quadrupole moment in our chosen gauge is

$$
I_{i j}^{\mathrm{ind}}=I_{i j}^{20}+I_{i j}^{22}+I_{i j}^{2-2}=\frac{32}{45} M^{6} \dot{\mathcal{E}}_{i j}^{\mathrm{ext}}
$$

which is proportional to the time derivative of the external tidal field-not the field itself as one would expect for Newtonian tidal couplings.

\footnotetext{
${ }^{2}$ Valid for all $\operatorname{Re} \ell>-1 / 2$.
} 
Lastly, we move from the LARF to the perturbed horizon and examine the effect of the static perturbation there. Hartle has shown [14] that to first order in the perturbation, the coordinate location of the event horizon of a slowly rotating black hole perturbed by a stationary distribution of matter is still at $r=2 M$. This is also true for a Schwarzschild black hole under static perturbations. Evaluating the full metric at $r=2 M$, we find the horizon metric is given by

$$
d s_{H}^{2}=4 M^{2}\left[1-2 \mu\left(M^{2} / b^{3}\right) P_{2}(\cos \theta)\right]\left(d \theta^{2}+\sin ^{2} \theta d \phi^{2}\right),
$$

where $P_{2}$ is the Legendre function. From this metric the scalar curvature of the horizon is obtained as

$$
\mathcal{R}=\frac{1}{2 M^{2}}\left[1-4 \mu\left(M^{2} / b^{3}\right) P_{2}(\cos \theta)\right] .
$$

So it is clear that the shape of the horizon does acquire a small quadrupolar component. But this deformation is not accompanied by an induced quadrupole moment in the LARF, at least in our chosen gauge.

\section{B. Ambiguity of the static induced quadrupole moment}

In the previous subsection, we found that a Schwarzschild black hole has a vanishing static induced quadrupole moment (SIQM) in response to the external

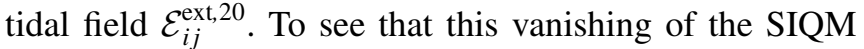
might possibly be a gauge effect, imagine replacing the radial coordinate $r$ in the expression $\Phi=(1 / 2) \mathcal{E}_{i j}^{\text {ext,20 }} n^{i} n^{j} r^{2}$ for the external tidal Newtonian potential by $r=\bar{r}(1+$ $\left.\chi M^{5} / \bar{r}^{5}\right)^{1 / 2}$, where $\chi$ is some dimensionless number of order unity. The result is $\Phi=(1 / 2) \mathcal{E}_{i j}^{\text {ext, } 20} n^{i} n^{j} \bar{r}^{2}+$ $(\chi / 2) M^{5} \mathcal{E}_{i j}^{\text {ext } 20} n^{i} n^{j} / \bar{r}^{3}$. By comparing this expression with Eq. (2) we read off a SIQM $I_{i j}=(\chi / 18) M^{5} \mathcal{E}_{i j}^{\text {ext, } 20} i j$. In Newtonian theory this procedure would obviously be naive, but in general relativity, where the unperturbed black hole metric can be expanded in powers of $M / r$ and the coefficients in that expansion depend on one's choice of radial coordinate and that choice is a "gauge" issue, this type of procedure is not obviously naive at all.

From our point of view, the best way to explore the gauge dependence of the SIQM is to ask whether it is physically measurable. If (as we shall find) physical mea- surements give a result that is ambiguous at some level, then that ambiguity constitutes a sort of gauge dependence of the SIQM.

In this section we shall study a thought experiment for measuring the SIQM, one based on coupling to a small, static external "test" octupole field $\mathcal{E}_{i j k}$ (proportional to the symmetrized and trace-removed gradient of some fiducial external quadrupolar tidal field). For simplicity we take $\mathcal{E}_{i j k}$ to be axisymmetric around the same $\mathrm{z}$ axis as

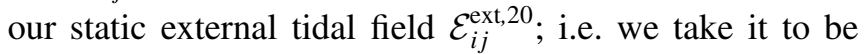
proportional to a tensor spherical harmonic of order $(\ell, m)=(3,0)$ :

$$
\mathcal{E}_{i j k} \sim \mathcal{Y}_{i j k}^{30} \text {. }
$$

The analysis in Ref. [29] says that, any SIQM $I_{i j}$


external octupole moment to produce a force that gradually changes the hole's momentum ${ }^{3}$ :

$$
\dot{P}^{i}=-\frac{1}{2} \mathcal{E}^{i}{ }_{j k} I_{j k} .
$$

(Equation (1.12) of Ref. [18]; Eq. (4b) of Ref. [29]). The same will be true if the central black hole is replaced by a neutron star or any other spherical body. The rate of change of momentum $\dot{P}^{i}$ can also be evaluated by a surface integral of the Landau-Lifshitz pseudotensor $t_{\mathrm{LL}}^{i j}$ in the LARF [18]:

$$
\dot{P}^{i}=-\oint(-g) t_{\mathrm{LL}}^{i j} d S_{j}
$$

Eqs. (36) and (37) for the coupling-induced force on the hole actually have ambiguities that arise from nonlinearities in the Einstein field equations. The origin of those ambiguities is discussed with care in Sec. I of Thorne and Hartle [18]. In this subsection we use Eq. (37) to calculate the force on the hole, and shall identify the ambiguities as those terms in which the force depends on the location of the integration surface. The result of our calculation will tell us, by comparison with Eq. (36), the SIQM and the amount by which it is ambiguous.

To compute the pseudotensor for insertion into Eq. (37), we must solve for the metric perturbation containing both the quadrupole and octupole terms:

$$
h_{a b}=h_{a b}^{(20)}+h_{a b}^{(30)}=\sum_{\ell=2,3} \operatorname{Diag}\left[(1-2 M / r) \hat{H}_{\ell}, \frac{\hat{H}_{\ell}}{1-2 M / r}, r^{2} \hat{K}_{\ell}, r^{2} \hat{K}_{\ell} \sin ^{2} \theta\right] Y^{\ell 0}(\theta, \phi) .
$$

When $\ell=2$, the general solution to $\hat{H}_{2}$ is given in Eq. (29). For $\ell=3$, we have

$$
\hat{H}_{3}(r)=\alpha_{3} P_{3}^{2}\left(\frac{r}{M}-1\right)+\beta_{3} Q_{3}^{2}\left(\frac{r}{M}-1\right) .
$$

\footnotetext{
${ }^{3}$ The test octupole field may also induce a static octupole moment $I_{i j k}$ in the central black hole, which will couple to the external quadrupolar tidal field. This coupling, as we shall show, contributes to the ambiguities in the definition of the SIQM.
} 
In order that both types of perturbation be finite at $r=2 M$, $\beta_{2}$ and $\beta_{3}$ must be set to zero [see Eq. (29)]. In order to deal with more general cases, however, we keep nonvanishing values for $\beta_{2}$ and $\beta_{3}$ in Eqs. (29) and (39) so the following analysis will be valid for stars as well as black holes. [For central bodies other than black holes, $\beta_{2}$ and $\alpha_{2}$ (and similarly $\beta_{3}$ and $\alpha_{3}$ ) are not independent of each other: $\beta_{2}$ is proportional to $\alpha_{2}$ with a proportionality constant that depends on the body's internal physical properties]. Having specified the metric perturbation, we then insert the full metric into the expression for the pseudotensor (Eq. (20.22) of Ref. [30])

$$
\begin{aligned}
(-g) t_{\mathrm{LL}}^{\alpha \beta}= & \frac{1}{16 \pi}\left\{\mathfrak{g}^{\alpha \beta}{ }_{, \lambda} \mathrm{g}^{\lambda \mu}{ }_{, \mu}-\mathrm{g}_{, \lambda}^{\alpha \lambda} \mathrm{g}^{\beta \mu}{ }_{, \mu}\right. \\
& +g_{\lambda \mu} g^{\nu \rho} \mathrm{g}^{\alpha \lambda}{ }_{, \nu} \mathrm{g}^{\beta \mu}{ }_{, \rho}-\left(g^{\alpha \lambda} g_{\mu \nu} \mathrm{g}^{\beta \nu}{ }_{, \rho} \mathrm{g}^{\mu \rho}{ }_{, \lambda}\right. \\
& \left.+g^{\beta \lambda} g_{\mu \nu} \mathrm{g}_{,{ }_{,}}^{\alpha \nu} \mathrm{g}^{\mu \rho}{ }_{, \lambda}\right)+\frac{1}{2} g^{\alpha \beta} g_{\lambda \mu} \mathfrak{g}_{, \rho}^{\lambda \nu} \mathfrak{g}^{\rho \mu}{ }_{, \nu} \\
& +\frac{1}{8}\left(2 g^{\alpha \lambda} g^{\beta \mu}-g^{\alpha \beta} g^{\lambda \mu}\right)\left(2 g_{\nu \rho} g_{\sigma \tau}\right. \\
& \left.\left.-g_{\rho \sigma} g_{\nu \tau}\right) \mathfrak{g}^{\nu \tau}{ }_{, \lambda} \mathfrak{g}^{\rho \sigma}{ }_{, \mu}\right\},
\end{aligned}
$$

and evaluate the surface integral at some radius $r=R$ in the LARF. Because of the axi-symmetry of the perturbed spacetime, only the $z$-component of $\dot{P}^{i}$ is nonzero. The result, up to first-order coupling and with uninteresting numerical coefficients being suppressed, has the following form:

$$
\begin{aligned}
\dot{P}^{z}= & \alpha_{3} \alpha_{2}\left[\frac{R^{4}}{M^{4}} \& \frac{R^{3}}{M^{3}} \& \frac{R^{2}}{M^{2}} \& \frac{R}{M} \& 1 \& \ldots\right] \\
& +\alpha_{3} \beta_{2}\left[1 \& \frac{M}{R} \& \ldots\right]+\beta_{3} \alpha_{2}\left[\frac{M^{3}}{R^{3}} \& \frac{M^{4}}{R^{4}} \& \ldots\right],
\end{aligned}
$$

where " $\&$ " is to be read "and a term of the order."

The constant terms in Eq. (41) [i.e., the "1"s] that are independent of the integration radius $R$ are the ones to be compared with Zhang's result (36) so as to deduced the gauge-invariant SIQM. Other terms that depend on $R$ constitute ambiguities ${ }^{4}$. Terms with positive power(s) of $R / M$ appear because the spacetime is not asymptotically flat, and they prevent us from minimizing the ambiguities by simply pushing the integration surface to infinity.

Let us step back and write down the most general form that the SIQM can take. By order of magnitude analysis of the response of any physical body (black hole, star, planet, moon,...) to a tidal field, we must have

\footnotetext{
${ }^{4}$ The $M^{2} / R^{2}$ term includes the effect of any octupole moment induced on the central body by the test octupole field. Note that $\dot{P}^{i}$ is a dimensionless vector. On dimensional grounds, the coupling between any induced octupole moment and the external tidal field must take the form $I^{i j k} \mathcal{E}_{j k} / R^{2}$ to contribute to $\dot{P}^{i}$. (Nonlinear coupling to the monopole moment can lead to similar terms that scale as higher, but not lower, powers of $1 / R$ ).
}

$$
I_{i j} \sim L^{5}(1+\xi) \mathcal{E}_{i j}^{\mathrm{ext}, 20} .
$$

Here $L$ is the size of the body ( $L \sim M$ for a black hole) and $\xi$ is a dimensionless number describing the SIQM's dependence on the integration radius $R-$ deviations from being well defined. From Eq. (31), we know the external tidal field scales as $\sim \alpha_{2} / M^{2}$. Similarly for the external octupole field, $\mathcal{E}_{i j k} \sim \alpha_{3} / M^{3}$. Using these relations, Eq. (36) becomes

$$
\dot{P}^{i} \sim L^{5}(1+\xi) \mathcal{E}_{j k}^{\mathrm{ext}, 20} \mathcal{E}^{i}{ }_{j k} \sim \alpha_{2} \alpha_{3}\left[\frac{L^{5}}{M^{5}}+\frac{\xi L^{5}}{M^{5}}\right] .
$$

Here the first term in the square bracket should be identified as the 1s in Eq. (41) [note again that $\beta_{2}$ and $\alpha_{2}$ are not independent of each other for stars]; and the second term should be identified as the sum of all $R$-dependent terms:

$$
\frac{\xi L^{5}}{M^{5}}=\frac{R^{4}}{M^{4}} \& \ldots \& \frac{R}{M} \& \frac{M}{R} \& \ldots
$$

In the case of a black hole we have $L \sim M$ and the smallest the right hand side of Eq. (44) can be is $\sim 1$ (for $R \sim M$ ), so $\xi \gtrsim 1$, i.e. the SIQM for a Schwarzschild black hole is ambiguous by an amount $\gtrsim M^{5} \mathcal{E}_{i j}^{\text {ext,20 }}$, i.e. totally ambiguous, since the largest we could expect $I_{i j}$ to be is $\sim M^{5} \mathcal{E}_{i j}^{\text {ext,20 }}$.

For central objects with $L \gg M$ (e.g., the Earth) we must choose $R>L$. The right hand side of Eq. (44) is then minimized by setting $R \simeq L$, giving $\xi \sim M / L \ll 1$ ( $\sim 10^{-9}$ in the case of the Earth) for the fractional ambiguities in the SIQM.

We comment that our result for a Schwarzschild black hole differs from what Suen has derived. Suen has given an unambiguous prescription to read out static multipole moments in non-asymptotically-flat spacetimes, which is based on transforming coordinates into a particular set of de Donder coordinates [31]. He has used his prescription to calculate the induced quadrupole moment of a Schwarzschild black hole when it is perturbed by a static, equatorial matter ring at large distances [19]. According to his prescription, the SIQM does not vanish. It is proportional to the tidal field produced by the ring:

$$
I_{i j}=-\frac{4}{21} M^{5} \mathcal{E}_{i j}^{\text {ring }} \text {. }
$$

The incompatibility between this result and the vanishing SIQM that we derived in Sec. IVA in Regge-Wheeler gauge and Schwarzschild coordinates illustrates the ambiguities of the SIQM. Both results, zero and $(-4 / 21) M^{5} \mathcal{E}_{i j}$ are less than or of order the ambiguity.

\section{THE TIDAL PHASE SHIFT}

In the LARF, the time-dependent induced quadrupole moment is $\pi / 4$ out of phase with the perturbing tidal field (Sec. III A). This large phase shift is quite different from 
the small phase lag angle, caused by viscous dissipation, between a planet's induced quadrupole moment and the perturbing tidal field. A closer black-hole analogy to a planet's viscous phase lag may be found by considering the tide raised on the hole's horizon by an orbiting moon.

In this section, we compute the tidal phase shift on the horizon for our perturbed Schwarzschild black hole. We will discuss in what sense it is and is not analogous to the fluid-planet's viscous phase lag. To calculate this phase shift, it is convenient to use the Newman-Penrose formalism [32] (see, e.g., chapter 1 of Ref. [33] for a review of the Newman-Penrose formalism). Appendix D summarizes some details of the Newman-Penrose formalism that are relevant for our purpose.

We consider two approaches to defining the tidal phase shift. In Sec. VA, we define the phase shift only in terms of quantities on the horizon (following the method suggested in Sec. VIIC of Ref. [22]), while in Sec. V B, we define a phase between the tide raised on the horizon and the "retarded" position of the moon (following the method used by Hartle in Ref. [15]).

\section{A. Phase of the tidal bulge on the horizon}

For Sec. V and Appendix D only, we use ingoing Eddington-Finkelstein coordinates $(\tilde{V}, r, \theta, \phi)$ and a $(+-$ $--)$ signature of the metric. The Schwarzschild metric in these coordinates is

$$
d s^{2}=\left(1-\frac{2 M}{r}\right) d \tilde{V}^{2}-2 d \tilde{V} d r-r^{2}\left(d \theta^{2}+\sin ^{2} \theta d \phi^{2}\right) .
$$

The ingoing Eddington-Finkelstein null time coordinate $\tilde{V}$ is related to the Schwarzschild time coordinate $t$ and radial coordinate $r$ by the following equation (Eq. (1b) of Box 32.2 of Ref. [30]):

$$
\tilde{V}=t+r^{*}=t+r+2 M \ln |r / 2 M-1|,
$$

and the Eddington-Finkelstein radial and angular coordinates $\{r, \theta, \phi\}$ are identical to those of Schwarzschild.

Our slowly orbiting moon deforms the Schwarzschild event horizon. By analogy with Newtonian tides, we would like to describe the horizon deformation as a perturbation that corotates (at a slightly different phase) with the tidal field that drives it. But this viewpoint inherently envisions the perturbed event horizon as a two-dimensional, evolving surface, rather than as a three-dimensional, global surface in spacetime. Therefore, before we can consider the phases of quantities on the horizon, we must first specify what we mean by time on the horizon.

Begin by considering the Schwarzschild event horizon (which is, of course, the three-surface $r=2 M$ ). There is a preferred way to slice the horizon into a single-parameter family of two-surfaces; this preferred slicing uses twosurfaces that are orthogonal to the Schwarzschild Killing vector $\partial_{t}=\partial_{\tilde{V}}$ that is timelike at the moon and null on the horizon. Following Hartle [15], we call this family of twosurfaces the "instantaneous horizon." The instantaneous horizon can be pictured as an evolving two-surface defined by $r=2 M$ and $\tilde{V}=$ const., so that $\tilde{V}$ plays the role of a "time" coordinate. Throughout this section, we use the terms "horizon" and "instantaneous horizon" interchangeably unless otherwise indicated.

We now consider how the horizon's perturbation evolves with time $\tilde{V}$. The moon's tidal field, characterized in the LARF by [Eq. (10)]

$$
\mathcal{E}_{i j}^{\mathrm{ext}}=\mathcal{E}_{i j}^{\mathrm{ext}, 20}+\mathcal{E}_{i j}^{\mathrm{ext}, 22}+\mathcal{E}_{i j}^{\mathrm{ext}, 2-2}
$$

deforms the otherwise spherical, static horizon. Because $\mathcal{E}_{i j}^{\text {ext,20 }}$ is static and axisymmetric, it cannot contribute to the phase shift. For the remaining tidal fields, $\mathcal{E}_{i j}^{\text {ext } 2 \pm 2}$, we shall consider only the $(2,2)$ mode in detail and the result for the $(2,-2)$ mode follows immediately.

On the horizon, it is the tangential-tangential components of the perturbing tidal field that drive the deformation (see, e.g., Eq. (6.80) of Ref. [22]); knowledge of these components is physically equivalent to knowledge of the Teukolsky function $\Psi_{0}$ [16] (see, e.g., Eq. (A7) of Ref. [34]). The Teukolsky function is a particular component of the Weyl tensor [Eq. (D5a)].

The horizon deformation is governed by the NewmanPenrose equation (Eq. (2.11) of Ref. [15])

$$
\left(\partial_{\tilde{V}}-2 \epsilon\right) \sigma^{(1)}=-2(i \Omega+\epsilon) \sigma^{(1)}=\Psi_{0}^{(1)} .
$$

This is also the "tidal force equation" (Eq. (6.80) of Ref. [22]). Here $\sigma=-\Sigma^{(1)}$ is a Newman-Penrose spin coefficient [Eq. (D3e)] and $\Sigma^{(1)}$ is the shear (i.e., the rate of change of the shape ${ }^{5}$ ) of the instantaneous horizon. Note that because $\Sigma$ and $\Psi_{0}$ vanish on the unperturbed instantaneous horizon, the spin coefficient $\epsilon$ takes its Schwarzschild value, which (in our tetrad) is the surface gravity of the instantaneous horizon $g_{H}=(4 M)^{-1}$.

Knowing $\Psi_{0}^{(1)}$, we can evaluate the horizon shear. Because $\Psi_{0}^{(1)}$ is first order in the perturbation, it may be evaluated on the horizon simply by letting $r$ go to $2 M$.

Beginning with the $(\ell, m)=(2,2)$ metric perturbation [Eq. (12), except we now choose the metric signature to be $(+---)$ ], we compute the perturbed Riemann tensor near the horizon and read off the component $\Psi_{0}^{(1)}$. The result is

\footnotetext{
${ }^{5}$ Recall that the shape of the instantaneous horizon (a twodimensional surface) is completely specified by its intrinsic scalar curvature $\mathcal{R}$.
} 


$$
\begin{aligned}
\Psi_{0}^{(1)}= & -i \sqrt{\frac{\pi}{5} \frac{\mu M \Omega}{b^{3}}}{ }_{2} Y^{22} e^{-2 i \Omega \tilde{V}+(8 / 3) M \Omega}+O\left(M^{2} \Omega^{2}\right) \\
= & \left|\Psi_{0}^{(1)}\right| \exp \left[2 i\left(\phi-\Omega \tilde{V}+\frac{4}{3} M \Omega-\frac{\pi}{4}\right)\right] \\
& +O\left(M^{2} \Omega^{2}\right) .
\end{aligned}
$$

Here ${ }_{2} Y^{22}$ is the spin-weighted spherical harmonic

$$
{ }_{2} Y^{22}=\frac{1}{2} \sqrt{\frac{5}{\pi}} \sin ^{4}\left(\frac{\theta}{2}\right) e^{2 i \phi} .
$$

With $\Psi_{0}^{(1)}$ in hand, we can calculate $\Sigma^{(1)}$ via Eq. (48). Inserting $\epsilon=2 g_{H}$ and $\omega=2 \Omega$ into Eq. (48) yields

$$
\begin{aligned}
\Sigma^{(1)}= & \frac{\Psi_{0}^{(1)}}{i \omega+2 \epsilon}=4 M \Psi_{0}^{(1)} e^{-2 i \Omega / g_{H}}+O\left(M^{2} \Omega^{2}\right) \\
= & \left|\Sigma^{(1)}\right| \exp \left[2 i\left(\phi-\Omega \tilde{V}+\frac{4}{3} M \Omega-\frac{\pi}{4}-\delta_{\text {Horizon }}\right)\right] \\
& +O\left(M^{2} \Omega^{2}\right) .
\end{aligned}
$$

where

$$
\delta_{\text {Horizon }} \equiv \Omega / g_{H}=4 M \Omega .
$$

The shear $\Sigma^{(1)}$ leads $\Psi_{0}^{(1)}$ (or equivalently, the perturbing tidal field at the horizon) by an angle $\delta_{\text {Horizon }}$. [Note that the first equality in Eq. (50) appears in Ref. [15] as Eq. (2.12).]

The shear is the time derivative of the shape. Therefore, the shape has a phase

$$
\mathcal{R}^{(1)}=\left|\mathcal{R}^{(1)}\right| \exp \left[2 i\left(\phi-\Omega \tilde{V}-\frac{8}{3} M \Omega\right)\right] .
$$

In other words, the shear leads the shape by $\pi / 4$.

The horizon phase shift in Eq. (51) follows directly from the tidal force Eq. (48). It is gauge-invariant since it only makes reference to gauge-invariant quantities measured on the instantaneous horizon. In Ref. [22] [Eq. (7.45), Fig. 57, and the surrounding discussion], an analogous horizon phase shift $\delta_{\text {Horizon }}^{\mathrm{H}}$ was deduced from the tidal force equation for a slowly rotating black hole perturbed by a stationary, axisymmetric tidal field-physically the same problem as Hartle studied [15]:

$$
\delta_{\text {Horizon }}^{\mathrm{H}}=\Omega_{H} / g_{H}=4 M \Omega_{H}=\left.\delta_{\text {Horzion }}\right|_{\Omega \rightarrow \Omega_{H}} .
$$

Here $\Omega_{H}$ is the horizon angular velocity.

Although Hartle also used the tidal force equation in his calculations, he chose to define the tidal phase shift in a different way and made his result gauge-invariant by making a connection between the angular positions on the horizon and angular positions at infinity through a null ray - a choice we will consider in detail in Sec. VB 1 and apply to our problem in Sec. V B 2.

The phase lead $\delta_{\text {Horizon }}$ is, in some ways, analogous to the phase shift of a tide raised on a nonrotating fluid planet. In the latter case, viscous dissipation causes the shape of the planet's surface to lag the normal-normal component of the perturbing tidal field by a small angle $\delta_{\text {visc }}$; somewhat analogously, the horizon shear leads the tangentialtangential component of the perturbing tidal field. Both phase shifts are small angles associated with dissipation (which manifests itself as a secular evolution of the energy and angular momentum of the moon's orbit). In the absence of dissipation, there is no phase shift. On the other hand, the phase shift $\delta_{\text {Horizon }}$ is a lead angle while $\delta_{\text {visc }}$ is a lag angle. Hartle explains this difference as a consequence of the teleological nature of the horizon [15]. Also as Hartle observed, when the angular velocity $\Omega$ is not small compared with $1 / M$, the deformation of the horizon cannot be described in terms of a phase shift [15].

\section{B. Phase shift between the tidal bulge and the moon}

As an alternative to the above way of defining the tidal phase shift, one can define it as the angle between the tidal bulge on the horizon and the location of the moon in its orbit. Hartle used this approach when he computed the tidal lead on a rotating hole perturbed by a stationary moon [15]. First, we will briefly summarize the aspects of Hartle's analysis which are relevant to our purpose. Then, we will apply his method to a slowly rotating moon around an otherwise Schwarzschild black hole.

\section{Tidal phase shift between a rotating horizon and stationary moon}

In Ref. [15], Hartle considers the problem of a distant, stationary moon perturbing a slowly rotating black hole.

The Kerr metric can be written as

$$
\begin{aligned}
d s^{2}= & \left(1-\frac{2 M r}{\Sigma}\right) d \tilde{V}^{2}-2 d \tilde{V} d r+\frac{4 a M r \sin ^{2} \theta}{\Sigma} d \tilde{V} d \tilde{\phi} \\
& +2 a \sin ^{2} \theta d r d \tilde{\phi}-\Sigma d \theta^{2} \\
& -\sin ^{2} \theta\left(a^{2}+r^{2}+\frac{2 a^{2} M r \sin ^{2} \theta}{\Sigma}\right) d \tilde{\phi}^{2}
\end{aligned}
$$

Here $\Sigma \equiv r^{2}+a^{2} \cos ^{2} \theta$. The coordinates $\tilde{V}$ and $\tilde{\phi}$ are related to the usual Boyer-Lindquist coordinates $t$ and $\phi$ by

$$
d t=d \tilde{V}-\frac{r^{2}+a^{2}}{\Delta} d r, \quad d \phi=d \tilde{\phi}-\frac{a}{\Delta} d r
$$

where $\Delta \equiv r^{2}-2 M r+a^{2}$. When $a=0$, Eq. (54) reduces to the Schwarzschild metric in Eddington-Finkelstein coordinates [Eq. (46)].

The event horizon is the surface $r=r_{+} \equiv M+$ $\sqrt{M^{2}-a^{2}}$. Just as in the Schwarzschild case considered above, the event horizon can be sliced into a singleparameter family of two-dimensional surfaces using the Killing vector $\partial_{\tilde{V}}$ which is timelike at infinity and null on the horizon. This family of surfaces is the instantaneous horizon. 
The distant moon raises a tidal bulge on the central hole's instantaneous horizon. In the limit that the moon is far away, the change in the horizon's shape (or equivalently, the change in the scalar curvature $\mathcal{R}$ of the instantaneous horizon), is purely quadrupolar.

The deformation is driven by the transverse-transverse component of the tidal field at the horizon, which is physically equivalent to the Teukolsky function, a particular component of the Riemann tensor $\Psi_{0}$ [Eq. (D5a)]. This component vanishes in the unperturbed Kerr spacetime [Eq. (D12a)], and the first order correction $\Psi_{0}^{(1)}$ has the form

$$
\Psi_{0}^{(1)}=S^{\ell m}(r)_{2} Y^{\ell m}(\theta, \tilde{\phi}),
$$

where ${ }_{2} Y^{\ell m}$ is a spin-weight-2 spherical harmonic. Because the perturbation is purely quadrupolar, we need only consider the case $\ell=2, m=2$ here, although Hartle considers the generic case. Hartle uses Teukolsky's solution [35] for the stationary radial functions $S^{\ell m}$ due to the $\ell$-pole perturbation caused by a distant, stationary point particle with mass $\mu$. Furthermore, while Hartle treats the case of a moon at any location $(\theta, \tilde{\phi})$, for concreteness we specify the moon's position as $(\theta, \tilde{\phi})=(\pi / 2,0)$. On the horizon, the Teukolsky function turns out to have the value (combining Eqs. (4.30), (4.31), (4.15), and (4.18) of Ref. [15])

$$
\begin{aligned}
\Psi_{0}^{(1), \mathrm{H}}= & \frac{i \mu M \Omega_{H}}{2 \sqrt{6} b^{3}} \sin ^{4}\left(\frac{\theta}{2}\right) \exp \left[2 i\left(\tilde{\phi}+2 M \Omega_{H}\right)\right] \\
& +O\left(\frac{M^{4}}{b^{4}}\right)+O\left(M^{2} \Omega_{H}^{2}\right) .
\end{aligned}
$$

The tidal field deforms the instantaneous horizon, changing its shape and thus its two-dimensional scalar curvature $\mathcal{R}$. Hartle computes the quadrupolar correction to the scalar curvature, $\mathcal{R}^{(1), \ell=2, \mathrm{H}}$, of the instantaneous horizon [Eq. (D9)]. His result is (Eqs. (4.26)-(4.27) of Ref. [15])

$$
\begin{aligned}
\mathcal{R}^{(1), \ell=2, \mathrm{H}} \propto & \cos \left[2\left(\tilde{\phi}+\frac{14}{3} M \Omega_{H}\right)\right]+O\left(\frac{M^{4}}{b^{4}}\right) \\
+ & O\left(M^{2} \Omega_{H}^{2}\right) .
\end{aligned}
$$

Instead of measuring the angle between the shear $\sigma$ and the tidal field $\Psi_{0}$ on the horizon, Hartle defines his phase lead as the angle between the shape and the moon's angular position. To make this definition gauge-invariant, Hartle chooses ingoing, zero-angular-monentum, null geodesics to be "lines of constant angle." He then compares the angular position of the horizon tidal bulge,

$$
\tilde{\phi}_{\text {bulge }}^{\mathrm{H}}=-\frac{14}{3} M \Omega_{H} \text {. }
$$

to the angular position of the moon on the horizon.

Consider stationary moon in the equatorial plane at (large) radius $r=b$ and at angular position $\phi=0$. An ingoing null ray, originating from the moon, intersects the instantaneous horizon at angular position ${ }^{6}$

$$
\tilde{\phi}_{\text {moon }}^{\mathrm{H}}=a / b-a / 2 M \text {. }
$$

The tidal bulge therefore leads the moon's position by an amount

$$
\delta_{\text {null map }}^{\mathrm{H}}=\tilde{\phi}_{\text {moon }}^{\mathrm{H}}-\tilde{\phi}_{\text {bulge }}^{\mathrm{H}}=\frac{8}{3} M \Omega_{H}+4 \frac{M^{2} \Omega_{H}}{b} .
$$

Here we have used the relation (valid for small $a / M$ ) that $a=4 M^{2} \Omega_{H}$, with $\Omega_{H}$ being the angular velocity of the hole. For simplicity, one can then take the limit $b \rightarrow \infty$.

Before continuing, we should remark that Hartle's prescription for constructing $\delta_{\text {null map }}^{\mathrm{H}}$ can be described without reference to the moon's position. Begin by computing the angular location of the tidal bulge on the horizon. Next, ingoing, zero-angular-momentum null rays from infinity define lines of constant angle, so that there is a one-to-one correspondence between angular positions on the horizon and angular positions at infinity. The angular position at infinity of the tidal bulge can thus be computed. Finally, perform the calculation again, but this time perturb a nonrotating spacetime; in this case, there will be no tidal friction. Because the Kerr and Schwarzschild spacetimes are asymptotically identical, one can unambiguously compare the angular position of the tidal bulge in the presence and in the absence of tidal friction: $\delta_{\text {null map }}^{\mathrm{H}}=\phi_{\text {bulge }}^{\mathrm{H}}-\phi_{\text {bulge,no friction }}^{\mathrm{H}}$. This is equivalent to the previous definition of $\delta_{\text {null map }}^{\mathrm{H}}$ provided that $b \rightarrow \infty$.

However, this alternative formulation of $\delta_{\text {null map }}^{\mathrm{H}}$ breaks down when the moon, not the horizon, rotates. The rotation is then described by $\Omega$, which is a parameter of the perturbation, not of the background spacetime. To eliminate tidal friction, one must let $\Omega \rightarrow 0$, which eliminates the perturbation ${ }^{7}$. Because of this failure, we prefer to consider Hartle's phase shift as a comparison of the position of the tidal bulge with the position of the moon.

\section{Tidal phase shift between a nonrotating horizon and rotating moon}

A similar analysis can be applied to our system, in which a distant moon in a slow, circular orbit raises a tide on a nonrotating black hole.

The moon orbits the central black hole along the world line specified by Eq. (8). In other words, the moon has a phase given by

\footnotetext{
${ }^{6}$ Note that there is a sign error in Hartle's analysis. Hartle incorrectly states that the ingoing null ray intersects the horizon at $+a / 2 M+O(a / b)$, not $-a / 2 M+O(a / b)$. Had we also made this error, there would be a coefficient of $20 / 3$ instead of $8 / 3$ in Eq. (61).

${ }^{7}$ Even if $\Omega \rightarrow 0$ resulted in a nonzero perturbation, it is unclear how to distinguish such a perturbation from a small change in the coordinates of the background spacetime.
} 


$$
\phi_{\text {moon }}(\tilde{V}) \equiv \Omega t=\Omega\left(\tilde{V}-b^{*}\right) .
$$

This must be compared with the location of the bulge on the hole's future horizon. Equation (52) for $\mathcal{R}^{(1)}$ [or, alternatively, inserting Eq. (49) into Eq. (D9)] shows that the tip of the tidal bulge has a phase given by

$$
\phi_{\text {bulge }}=\Omega \tilde{V}+\frac{8}{3} M \Omega .
$$

As time $\tilde{V}$ passes, this bulge rotates around and around the horizon, with the same angular velocity $\Omega$ as the moon that raises the tide.

Following Hartle, we compare the angular location of the tidal bulge, $\phi_{\text {bulge }}(\tilde{V})$, with the angular location of the moon, $\phi_{\text {moon }}(\tilde{V})$, using ingoing, zero angular momentum (ZAM) null rays to provide the connection between $\phi$ at the moon's orbit and $\phi$ on the horizon. In the ingoing Eddington-Finkelstein coordinates that we are using, these ZAM rays have a very simple form:

$$
\{\tilde{V}, \theta, \phi\}=\text { const., } \quad r \text { decreases from } b \text { to } 2 M \text {. }
$$

Since $\tilde{V}, \theta$, and $\phi$ are all constant along these rays, they give us a one-to-one map of events $\{\tilde{V}, r=b, \theta, \phi\}$ at the moon's orbital radius to events $\{\tilde{V}, r=2 M, \theta, \phi\}$ on the horizon that have identically the same $\tilde{V}, \theta$, and $\phi$. With the aid of this map, we conclude that the angle by which the horizon bulge lags the moon's position is

$$
\delta_{\text {null map }} \equiv \phi_{\text {bulge }}(\tilde{V})-\phi_{\text {moon }}(\tilde{V})=\frac{8}{3} M \Omega+\Omega b^{*} .
$$

[Eqs. (62) and (63)]. Again, the phase shift is a phase lead, not a phase lag, due to the teleological nature of the horizon.

In addition to the teleological phase shift of order $M \Omega$, $\delta_{\text {null map }}$ contains a much larger term of magnitude $\Omega b^{*}$; this term reflects the choice to use an ingoing-null-ray mapping between the moon and the horizon. A similar term appears in Hartle's calculation [Eq. (61)], but in Hartle's system the term is much smaller than the teleological phase shift size (specifically, smaller by a factor of $M / b)$, whereas $\Omega b^{*} \gg M \Omega$.

One could avoid this problem by defining the phase shift to include only terms of order $M \Omega$ and $M \Omega_{H}$. With this definition, the remaining tidal phase leads are the same: $(8 / 3) M \Omega$, as one would expect, given that a there should be no tidal shift at all if the moon were to rotate at the hole's angular velocity, i.e., if $\Omega=\Omega_{H}$.

We prefer, however, to define the tidal lead angle in as the angle $\delta_{\text {Horizon }}=4 M \Omega$ by which the horizon shear leads the horizon tidal field. This angle, in contrast to $\delta_{\text {null map }}$, is defined in terms of an "instantaneous" (spacelike) connection between the moon and the horizon, i.e., by the near zone mapping of the moon's position to the horizon tidal field's $\left[\Psi_{0}^{(1)}\right.$,s] maximum. [Had the moon been in the radiation zone $(b \gg \lambda / 2 \pi)$, one would have expected the connection to be lightlike.]

\section{CONCLUSION}

For our simple system of a Schwarzschild black hole and circularly orbiting moon, we have found that the timedependent part of the moon's tidal field induces a quadrupole moment that is unambiguous. The static induced quadrupole moment was found to be zero in the ReggeWheeler gauge, but it is ambiguous in general. The ambiguity of the static induced quadrupole moment leads to an ambiguity in the phase of the induced quadrupole moment in the LARF; however, the tidal bulge on the horizon still has a well-defined phase shift with respect to the orbiting moon. Because of the ambiguity of the induced quadrupole moment and the LARF phase shift, we conclude that the polarizability and phase shift are not suitable for constructing a body-independent description of tidal coupling in EMRIs.

However, this conclusion does not eliminate the possibility of developing a body-independent language to describe tidal coupling, including cases where the central body is a black hole. It might be possible, for instance, to define a new set of induced "dissipative multipole moments" for the central body-i.e. moments that vanish in the absence of tidal friction. Such dissipative moments would still be linear in the perturbing tidal field, so one could still define a polarizability. Also, by ignoring any nondissipative tidal coupling, the phase shift might no longer contain additional information. Even if such an extension does not prove feasible, tidal coupling can still be described in the more conventional (but still bodyindependent) language of energy and angular momentum transfer between the moon and the central body.

Other future work could include generalizing our analysis to spinning black holes, treating noncircular, nonequatorial orbits, and (most importantly) studying how information about tidal coupling in EMRIs can be extracted from the gravitational waves detected by LISA.

\section{APPENDIX A: SYMMETRIC TRACE-FREE TENSOR NOTATION FOR SPHERICAL HARMONICS}

The scalar spherical harmonics $Y^{\ell m}(\theta, \phi)$ can be written in terms of of rank- $\ell$ symmetric trace-free (STF) tensors [23]. The spherical harmonics $Y^{2 m}(\theta, \phi)$ that have been used in this paper are

$$
\begin{aligned}
Y^{2 \pm 2}(\theta, \phi) & =\frac{1}{4} \sqrt{\frac{15}{2 \pi}} \sin ^{2} \theta e^{ \pm 2 i \phi} \\
Y^{20}(\theta, \phi) & =\frac{1}{8} \sqrt{\frac{5}{\pi}}(1+3 \cos 2 \theta) .
\end{aligned}
$$

They can be written in terms of rank-2 STF tensors as 
(Eq. (2.11) of Ref. [23])

$$
Y^{2 m}(\theta, \phi)=\mathcal{Y}_{i j}^{2 m} n^{i} n^{j},
$$

where $n^{i} \equiv x^{i} / r$ and $\mathcal{Y}_{i j}^{2 m}$ are the STF tensors given by (Eq. (2.12) of Ref. [23]):

$$
\begin{aligned}
& \mathcal{Y}_{i j}^{20}=-\frac{1}{4} \sqrt{\frac{5}{\pi}}\left(\begin{array}{ccc}
1 & 0 & 0 \\
0 & 1 & 0 \\
0 & 0 & -2
\end{array}\right) \\
& \mathcal{Y}_{i j}^{2 \pm 2}=\frac{1}{4} \sqrt{\frac{15}{2 \pi}}\left(\begin{array}{ccc}
1 & \pm i & 0 \\
\pm i & -1 & 0 \\
0 & 0 & 0
\end{array}\right)
\end{aligned}
$$

\section{APPENDIX B: TIME-DEPENDENT PERTURBATION EQUATIONS}

In Regge-Wheeler gauge, the metric perturbation for a given even-parity $(\ell, m, \omega)$ mode depends on the three radial functions $H, H_{1}$, and $K$. In this appendix, we introduce the Zerilli function $\mathbb{Z}$ and the Regge-Wheeler function $\mathbb{X}$ and describe how we obtain the radial functions from them. The description here will hold for a general $(\ell, m, \omega)$, while the results derived in Sec. III rely on the special case when $(\ell, m, \omega)=(2,2,2 \Omega)$.

The original Zerilli's master function is defined implicitly through its relation with the two radial functions $H_{1}$ and $K$ [Eqs. (13) and (14) of Ref. [25] with $R_{L M}^{(e)}$ replaced by $\mathbb{Z}]$ :

$$
\begin{gathered}
H_{1}=-i \omega \frac{\lambda r^{2}-3 \lambda M r-3 M^{2}}{(r-2 M)(\lambda r+3 M)} \mathbb{Z}-i \omega r \frac{d \mathbb{Z}}{d r}, \\
K=\frac{\lambda(\lambda+1) r^{2}+3 \lambda M r+6 M^{2}}{r^{2}(\lambda r+3 M)} \mathbb{Z}+\frac{d \mathbb{Z}}{d r^{*}},
\end{gathered}
$$

where

$$
\lambda \equiv \frac{1}{2}(\ell-1)(\ell+2) .
$$

Using the algebraic relationship (Eq. (10) of Ref. [26])

$$
\begin{aligned}
\left(\frac{3 M}{r}+\lambda\right) H= & {\left[i \omega r-\frac{i(\lambda+1) M}{\omega r^{2}}\right] H_{1} } \\
& +\left(\lambda+\frac{M}{r}-\frac{M^{2} / r^{2}+\omega^{2} r^{2}}{1-2 M / r}\right) K
\end{aligned}
$$

one can obtain $H$ in terms of the Zerilli function

$$
H=\left[\frac{\omega^{2} r^{2}}{2 M-r}+\frac{s_{1}}{r^{2}(3 M+\lambda r)^{2}}\right] \mathbb{Z}+s_{2} \frac{d \mathbb{Z}}{d r},
$$

in which

$$
\begin{aligned}
& s_{1}=9 M^{2}(M+\lambda r)+\lambda^{2} r^{2}[3 M+(\lambda+1) r], \\
& s_{2}=\frac{-3 M^{2}-3 \lambda M r+\lambda r^{2}}{r(3 M+\lambda r)} .
\end{aligned}
$$

The Zerilli function obeys the wave equation (Eqs. (18) and (19) of Ref. [25]):

$$
\left[\frac{d^{2}}{d r^{* 2}}+\omega^{2}-V(r)\right] \mathbb{Z}=0
$$

in which the potential term is given by

$$
\begin{aligned}
V(r)= & \frac{2(r-2 M)}{r^{4}(\lambda r+3 M)^{2}}\left[\lambda^{2}(\lambda+1) r^{3}+3 \lambda^{2} M r^{2}\right. \\
& \left.+9 \lambda M^{2} r+9 M^{3}\right] .
\end{aligned}
$$

The odd-parity master function, the Regge-Wheeler function, is defined in Eq. (23) of Ref. [24] (and is called $Q$ in Regge and Wheeler's notation). It obeys the differential equation (Eq. (7) of Ref. [26]):

$$
\left[\frac{d^{2}}{d r^{* 2}}+\omega^{2}-\left(1-\frac{2 M}{r}\right)\left(\frac{\ell(\ell+1)}{r^{2}}-\frac{6 M}{r^{3}}\right)\right] \mathbb{X}=0 .
$$

The connection between the Regge-Wheeler and Zerilli functions was first found by Chandrasekhar and is listed, e.g., in Eq. (152) of Ch. 4 of Ref. [33]:

$$
\begin{aligned}
{[\lambda(\lambda+1)-3 i M \omega] \mathbb{Z}=} & {\left[\lambda(\lambda+1)+\frac{9 M^{2}(r-2 M)}{r^{2}(\lambda r+3 M)}\right] \mathbb{X} } \\
& +3 M\left(1-\frac{2 M}{r}\right) \frac{d \mathbb{X}}{d r} .
\end{aligned}
$$

This completes our metric reconstruction scheme from the Regge-Wheeler function. We are now ready to evaluate the radial metric perturbation functions $H, H_{1}$, and $K$ for the $(\ell, m)=(2,2)$ mode of the perturbations. Expanding $X^{H}$ [given in Eq. (16) in powers of $\tilde{r} \equiv r / 2 M$ to first order in $\varepsilon \equiv 2 M \omega$, we obtain

$$
X^{H}=\mathcal{A}\left[\left(1+\frac{13}{12} i \varepsilon\right) \tilde{r}^{3}+\sum_{n=5}^{\infty} \frac{i \varepsilon}{n \tilde{r}^{n-3}}+O\left(\varepsilon^{2}\right)\right] .
$$

Here $\mathcal{A}$ is an overall scaling factor (Eq. (20) in Sec. III A). While the summation can be rewritten as a closed-form expression, we prefer to stay in the series notation, since our interest is in reading various powers of $r$ in the resulting first-order tidal field. Equation (B7) is the value of the Regge-Wheeler function in the LARF; inserting it into Eq. (B6) yields the expression for $\mathbb{Z}$ in the LARF [We shall suppress " $O\left(\varepsilon^{2}\right)$ " hereafter]: 


$$
\begin{aligned}
\mathbb{Z}= & \mathcal{A}\left(1+\frac{4 i \varepsilon}{3}\right)\left[\tilde{r}^{3}+\frac{3 \tilde{r}^{2}}{4}-\frac{9 \tilde{r}}{16}-\frac{21}{64}+\frac{63}{256 \tilde{r}}\right] \\
& +\mathcal{A}\left[\frac{-945-236 i \varepsilon}{5120 \tilde{r}^{2}}+\frac{8505+15436 i \varepsilon}{61440 \tilde{r}^{3}}\right. \\
& \left.+O\left(\frac{1}{\tilde{r}^{4}}\right)\right] .
\end{aligned}
$$

Inserting $\mathbb{Z}$ into Eqs. (B1)-(B3) yields $H_{1}, K$, and $H$. Expanded in powers of $\tilde{r}$ and to first order in $\varepsilon$, these radial functions are given by

$$
\begin{aligned}
H= & \frac{A}{M}\left[(3+4 i \varepsilon)\left(\tilde{r}^{2}-\tilde{r}\right)+\frac{i \varepsilon}{10 \tilde{r}^{3}}+\frac{3 i \varepsilon}{20 \tilde{r}^{4}}\right]+O\left(\tilde{r}^{-5}\right), \\
H_{1}= & \frac{i A \varepsilon}{4 M}\left[-8 \tilde{r}^{3}-2 \tilde{r}^{2}+4 \tilde{r}+1+\tilde{r}^{-1}+\tilde{r}^{-2}+\tilde{r}^{-3}\right. \\
& \left.+\tilde{r}^{-4}\right]+O\left(\tilde{r}^{-5}\right), \\
K= & \frac{A}{M}\left[(3+4 i \varepsilon)\left(\tilde{r}^{2}-\frac{1}{2}\right)+\frac{i \varepsilon}{10 \tilde{r}^{3}}+\frac{i \varepsilon}{8 \tilde{r}^{4}}+\frac{9 i \varepsilon}{70 \tilde{r}^{5}}+\frac{i \varepsilon}{8 \tilde{r}^{6}}\right] \\
& +O\left(\tilde{r}^{-7}\right)
\end{aligned}
$$

\section{APPENDIX C: TIME-INDEPENDENT PERTURBATION EQUATIONS}

As is evident from the time-dependent perturbation theory, as $\omega \rightarrow 0, H_{1}$ goes to zero. In the static case, then, there are only two radial functions, $\hat{H}$ and $\hat{K}$ (where the hats signify that they represent the time-independent perturbations). Specializing to the axisymmetric case, the metric perturbation is

$$
\begin{aligned}
h_{a b}^{(\ell 0)}= & \operatorname{Diag}\left[(1-2 M / r) \hat{H}, \frac{\hat{H}}{1-2 M / r}, r^{2} \hat{K}, r^{2} \hat{K} \sin ^{2} \theta\right] \\
& \times Y^{\ell 0}(\theta, \phi) .
\end{aligned}
$$

The linearized Einstein equations governing $\hat{H}$ and $\hat{K}$ are given in Eqs. (9d) and (9e) of Ref. [26] with $H_{1}=0$ and $\omega=0$ ( $k=0$ in Edelstein and Vishveshwara's notation):

$$
\begin{gathered}
\frac{d \hat{K}}{d r}=\frac{d \hat{H}}{d r}+\frac{2 M}{r^{2}}\left(1-\frac{2 M}{r}\right)^{-1} \hat{H}, \\
\frac{2 M}{r^{2}} \frac{d \hat{K}}{d r}=\left(1-\frac{2 M}{r}\right) \frac{d^{2} \hat{H}}{d r^{2}}+\frac{2}{r} \frac{d \hat{H}}{d r}-\frac{\ell(\ell+1)}{r^{2}} \hat{H} .
\end{gathered}
$$

Eliminating $d \hat{K} / d r$ from these two equations, we can then write a single second-order differential equation for $H$ in terms of the variable $z \equiv r / M-1$ (same as Eq. (4.9) of Ref. [14]):

$$
\left(1-z^{2}\right) \frac{d^{2} \hat{H}}{d z^{2}}-2 z \frac{d \hat{H}}{d z}+\left[\ell(\ell+1)-\frac{4}{1-z^{2}}\right] \hat{H}=0 .
$$

This takes a form of the associated Legendre differential equation. The general solution for $\hat{H}$ is therefore

$$
\hat{H}=\alpha_{\ell} P_{\ell}^{2}(r / M-1)+\beta_{\ell} Q_{\ell}^{2}(r / M-1) .
$$

With the general solution for $\hat{H}$, we can integrate Eq. (C1) or (C2) to find $\hat{K}$. For $\ell=2$, we have

$$
\begin{aligned}
\hat{K}_{2}(r)= & {\left[\alpha_{2} P_{2}^{1}(r / M-1)+\beta_{2} Q_{2}^{1}(r / M-1)\right] } \\
& \times \frac{2 M}{\sqrt{r(r-2 M)}}+\hat{H}_{2}(r) .
\end{aligned}
$$

\section{APPENDIX D: NEWMAN-PENROSE FORMALISM}

In this appendix, we summarize some equations of the Newman-Penrose formalism for our choice of tetrad. In this Appendix and in Sec. V only, we use ingoing Eddington-Finkelstein coordinates $(\tilde{V}, r, \theta, \phi)$ and a $(+-$ $--)$ signature of the metric.

\section{Newman-Penrose quantities for Schwarzschild}

We adopt the Hartle's null tetrad, which is given by Eqs. (4.2) of Ref. [15], together with the normalization conditions $\ell^{\mu} n_{\mu}=1$ and $m^{\mu} \bar{m}_{\mu}=-1$. The tetrad vectors have components [using the notation $e^{\mu}=\left(e^{\tilde{V}}, e^{r}, e^{\theta}, e^{\phi}\right)$ ]

$$
\begin{aligned}
\ell^{\mu} & =\left(1, \frac{1}{2}-\frac{M}{r}, 0,0\right), \\
n^{\mu} & =(0,-1,0,0), \\
m^{\mu} & =\left(0,0, \frac{1}{\sqrt{2} r}, \frac{i}{\sqrt{2} r \sin \theta}\right), \\
\bar{m}^{\mu} & =\left(0,0, \frac{1}{\sqrt{2} r},-\frac{i}{\sqrt{2} r \sin \theta}\right) .
\end{aligned}
$$

Note that throughout this Appendix, an overbar denotes complex conjugation.

From these basis vectors, we define the direction derivatives

$$
\begin{gathered}
D=\ell^{\mu} \partial_{\mu}, \quad \Delta=n^{\mu} \partial_{\mu}, \quad \delta=m^{\mu} \partial_{\mu}, \\
\text { and } \quad \bar{\delta}=\bar{m}^{\mu} \partial_{\mu} .
\end{gathered}
$$

Our conventions for the spin coefficients follow Ref. [15] [specifically, Eqs. (2.2) and (2.3)]. The spin coefficients are defined by 


$$
\begin{aligned}
\kappa & =\ell_{\mu ; \nu} m^{\mu} \ell^{\nu}, \\
\pi & =-n_{\mu ; \nu} \bar{m}^{\mu} \ell^{\nu}, \\
\rho & =\ell_{\mu ; \nu} m^{\mu} \bar{m}^{\nu}, \\
\mu & =-n_{\mu ; \nu} \bar{m}^{\mu} m^{\nu}, \\
\sigma & =\ell_{\mu ; \nu} m^{\mu} m^{\nu}, \\
\lambda & =-n_{\mu ; \nu} \bar{m}^{\mu} \bar{m}^{\nu}, \\
\epsilon & =\frac{1}{2}\left(\ell_{\mu ; \nu} n^{\mu} \ell^{\nu}-m_{\mu ; \nu} \bar{m}^{\mu} \ell^{\nu}\right), \\
\alpha & =\frac{1}{2}\left(\ell_{\mu ; \nu} n^{\mu} \bar{m}^{\nu}-m_{\mu ; \nu} \bar{m}^{\mu} \bar{m}^{\nu}\right), \\
\beta & =\frac{1}{2}\left(\ell_{\mu ; \nu} n^{\mu} m^{\nu}-m_{\mu ; \nu} \bar{m}^{\mu} m^{\nu}\right) .
\end{aligned}
$$

The spin coefficients for the Schwarzschild spacetime are

$$
\begin{aligned}
\kappa & =\sigma=\lambda=\nu=\tau=\pi=\gamma=0, \\
\epsilon & =\frac{M}{2 r^{2}} \\
\rho & =-\frac{r-2 M}{2 r^{2}} \\
\mu & =-\frac{1}{r} \\
\alpha & =-\beta=-\frac{1}{2 \sqrt{2} r \tan \theta} .
\end{aligned}
$$

Because we are only interested in vacuum regions of spacetime, the Riemann and Weyl tensors are interchangeable. The Weyl components are defined in vacuum by

$$
\begin{aligned}
& \Psi_{0}=-R_{\alpha \beta \gamma \delta} \ell^{\alpha} m^{\beta} \ell^{\gamma} m^{\delta}, \\
& \Psi_{1}=-R_{\alpha \beta \gamma \delta} \ell^{\alpha} n^{\beta} \ell^{\gamma} m^{\delta}, \\
& \Psi_{2}=-\frac{1}{2} R_{\alpha \beta \gamma \delta}\left(\ell^{\alpha} n^{\beta} \ell^{\gamma} n^{\delta}+\ell^{\alpha} n^{\beta} m^{\gamma} \bar{m}^{\delta}\right), \\
& \Psi_{3}=-R_{\alpha \beta \gamma \delta} \ell^{\alpha} n^{\beta} \bar{m}^{\gamma} n^{\delta}, \\
& \Psi_{4}=-R_{\alpha \beta \gamma \delta} n^{\alpha} \bar{m}^{\beta} n^{\gamma} \bar{m}^{\delta} .
\end{aligned}
$$

Their values for the Schwarzschild spacetime are

$$
\begin{aligned}
& \Psi_{0}=\Psi_{1}=\Psi_{3}=\Psi_{4}=0, \\
& \Psi_{2}=-\frac{M}{r^{3}} .
\end{aligned}
$$

$$
\begin{aligned}
\ell^{\mu} & =\left(1, \frac{r^{2}-2 M r+a^{2}}{2\left(r^{2}+a^{2}\right)}, 0, \frac{a}{r^{2}+a^{2}}\right), \\
n^{\mu} & =\left(0,-\frac{2\left(a^{2}+r^{2}\right)}{2 r^{2}+a^{2}+a^{2} \cos 2 \theta}, 0,0\right)+\frac{-a^{2}+a^{2} \cos 2 \theta}{2\left(a^{2}+2 r^{2}+a^{2} \cos 2 \theta\right)} \ell^{\mu}+\frac{-a \sin \theta}{\sqrt{2}(i r+a \cos \theta)} m^{\mu}+\frac{-a \sin \theta}{\sqrt{2}(-i r+a \cos \theta)} \bar{m}^{\mu}, \\
m^{\mu} & =\left(0,-\frac{a \sin \theta\left(r^{2}-2 M r+a^{2}\right)}{2 \sqrt{2}\left(r^{2}+a^{2}\right)(-i r+a \cos \theta)}, \frac{1}{\sqrt{2}(r+i a \cos \theta)}, \frac{(i r+a \cos \theta) \csc \theta}{\sqrt{2}\left(r^{2}+a^{2}\right)}\right), \\
\bar{m}^{\mu} & =\left(0,-\frac{a \sin \theta\left(r^{2}-2 M r+a^{2}\right)}{2 \sqrt{2}\left(r^{2}+a^{2}\right)(i r+a \cos \theta)}, \frac{1}{\sqrt{2}(r-i a \cos \theta)}, \frac{(-i r+a \cos \theta) \csc \theta}{\sqrt{2}\left(r^{2}+a^{2}\right)}\right) .
\end{aligned}
$$


Then, one can compute the spin coefficients for this tetrad from Eqs. (D3a)-(D3i):

$$
\begin{aligned}
\kappa & =\sigma=0, \\
\lambda & =O\left(a^{2}\right), \\
\nu & =O\left(a^{2}\right), \\
\tau & =\frac{-i(2 M+r) \sin \theta a}{2 \sqrt{2} r^{3}}+O\left(a^{2}\right), \\
\pi & =\frac{i(4 M+r) \sin \theta a}{2 \sqrt{2} r^{3}}+O\left(a^{2}\right), \\
\gamma & =\frac{-i \cos \theta a}{2 r^{2}}+O\left(a^{2}\right), \\
\epsilon & =\frac{M}{2} \frac{r^{2}-a^{2}}{\left(r^{2}+a^{2}\right)^{2}}=\frac{M}{2 r^{2}}+O\left(a^{2}\right), \\
\rho & =-\frac{r-2 M}{2 r^{2}}-\frac{i(r-2 M) \cos \theta a}{2 r^{3}}+O\left(a^{2}\right), \\
\mu & =-\frac{1}{r}+O\left(a^{2}\right), \\
\alpha & \left.=\frac{-\cot \theta}{2 \sqrt{2} r}-\frac{i[-3 M+(3 M+2 r) \cos 2 \theta] a}{4 \sqrt{2} r^{3} \sin \theta}+O(a \oplus), 11 \mathrm{j}\right) \\
\beta & =\frac{\cot \theta}{2 \sqrt{2} r}-\frac{i[M+r+(r-M) \cos 2 \theta] a}{4 \sqrt{2} r^{3} \sin \theta}+O\left(a^{2}\right)(\mathrm{D} 11 \mathrm{k})
\end{aligned}
$$

The directional derivatives are then given by Eq. (D2).

Using the Kerr metric [Eq. (54)] and Hartle's choice for the tetrad [Eqs. (D10a)-(D10d)], one can compute the curvature for Kerr and read off the curvature scalars via Eqs. (D5a)-(D5e):

$$
\begin{aligned}
& \Psi_{0}=\Psi_{1}=0, \\
& \Psi_{2}=-\frac{M}{(r-i a \cos \theta)^{3}}, \\
& \Psi_{3}=-\frac{3 i a M \sin \theta}{\sqrt{2}(r-i a \cos \theta)^{4}}, \\
& \Psi_{4}=\frac{3 i a^{2} M \sin ^{2} \theta}{(i r+a \cos \theta)^{5}} .
\end{aligned}
$$

The tidal force Eq. (D8) relates $\Psi_{0}^{(1)}$ to $\sigma^{(1)}$. The correction to the scalar curvature of the horizon, $\mathcal{R}^{(1)}$, is given by Eq. (D9).

For a stationary moon perturbing a slowly rotating Kerr black hole, the frequency of the perturbation is $\omega=$ $-2 \Omega_{H}$.

\section{ACKNOWLEDGMENTS}

We are grateful to Kip Thorne for suggesting this problem and for his encouragement and advice, and to Yasushi Mino for helpful discussions. This research was supported in part by NASA Grants No. NAG5-12834 and No. NAG510707 and NSF Grant No. PNY-0099568. We used MATHEMATICA and MAPLE to verify some of the equations in this paper.
[1] A. Abramovici et al., Science 256, 325 (1992).

[2] K. S. Thorne, in Proceedings of the Snowmass 94 Summer Study on Particle and Nuclear Astrophysics and Cosmology, edited by E. Kolb and R. Peccei (World Scientific, Singapore, 1995), p. 398.

[3] F. D. Ryan, Phys. Rev. D 52, 5707 (1995).

[4] The word bothrodesy was coined by Sterl Phinney, and he first used it in a seminar at Caltech on June 8, 2001.

[5] E. S. Phinney, LISA Science Requirements, Proceedings of the LISA International Science Team [LIST], 2001, available at the web site of LIST Working Group 1: http:// www.tapir.caltech.edu/ listwg1/.

[6] J. R. Gair et al., Classical Quantum Gravity 21, S1595 (2004).

[7] J. R. Gair et al., in GW-DAW proceedings (2004b), available online at http://arxiv.org/abs/gr-qc/0405137.

[8] L. S. Finn and K. S. Thorne, Phys. Rev. D 62, 124021 (2000).

[9] K. S. Thorne, in The Future of Theoretical Physics and Cosmology, edited by G. Gibbons, E. P. S. Shellard, and S. J. Rankin (Cambridge University Press, Cambridge, England, 2003), Chap. 5.

[10] K. S. Thorne (private communication).
[11] M. Colpi, S. L. Shapiro, and I. Wasserman, Phys. Rev. Lett. 57, 2485 (1986).

[12] F. D. Ryan, Phys. Rev. D 55, 6081 (1997).

[13] T. D. Lee, Phys. Rev. D 35, 3637 (1987).

[14] J. B. Hartle, Phys. Rev. D 8, 1010 (1973).

[15] J. B. Hartle, Phys. Rev. D 9, 2749 (1974).

[16] S. A. Teukolsky, Phys. Rev. Lett. 29, 1114 (1972).

[17] G. H. Darwin, Phil. Trans. R. Soc. London 170, 1 (1879).

[18] K. S. Thorne and J.B. Hartle, Phys. Rev. D 31, 1815 (1985).

[19] W. M. Suen, Phys. Rev. D 34, 3633 (1986).

[20] We follow the same notation as in Ref. [23].

[21] E. Poisson, Phys. Rev. D 70, 084044 (2004).

[22] K. S. Thorne, R. Price, and D. MacDonald, Black Holes: The Membrane Paradigm (Yale University Press, New Haven, 1986).

[23] K. S. Thorne, Rev. Mod. Phys. 52, 299 (1980).

[24] T. Regge and J. A. Wheeler, Phys. Rev. 108, 1063 (1957).

[25] F. J. Zerilli, Phys. Rev. D 2, 2141 (1970).

[26] L. Edelstein and C. V. Vishveshwara, Phys. Rev. D 1, 3514 (1970).

[27] E. Poisson and M. Sasaki, Phys. Rev. D 51, 5753 (1995).

[28] A. Erdélyi et al., Higher Transcendental Functions, Vol. I 
(McGraw-Hill, New York, 1953).

[29] X.-H. Zhang, Phys. Rev. D 31, 3130 (1985).

[30] C. Misner, K. S. Thorne, and J. A. Wheeler, Gravitation (W. H. Freeman and Company, New York, 1973).

[31] W. M. Suen, Phys. Rev. D 34, 3617 (1986).

[32] E. Newman and R. Penrose, J. Math. Phys. (N.Y.) 3, 566 (1962).
[33] S. Chandrasekhar, The Mathematical Theory of Black Holes (Oxford University Press, Oxford, 1985).

[34] R. H. Price and K. S. Thorne, Phys. Rev. D 33, 915 (1986).

[35] S. Teukolsky, Ph.D. thesis, Caltech, 1973 (unpublished).

[36] S. Kobayashi and K. Nomizu, Foundations of Differential Geometry, Vol. II (Interscience, New York, 1963). 